\title{
Michael Cusanovich: a man of many talents and interests
}

Terry Meyer

Published online: 22 April 2011

(C) Springer Science+Business Media B.V. 2011

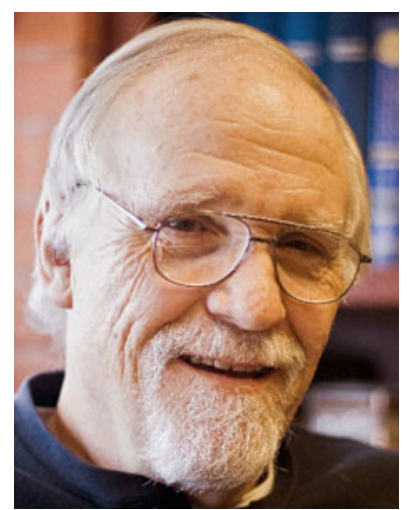

Michael Cusanovich, 1942-2010

How does one perform two or more independent tasks, each crucial and time-constrained, simultaneously? That was usual with Mike. He was often solving scientific, technical, and administrative problems with colleagues on the phone while working on his dual-screened computer, one for the project at hand and the other for his daily schedule. To us, there were seemingly not enough hours in the day to do all the work for which he volunteered. His solution was to sleep less. He would typically come into the lab about $6 \mathrm{AM}$, working at his computer and leaving for his first meeting at about 7 or 8 . As the quintessential problem solver, there would be a succession of meetings with faculty, staff, and students and between, he would be writing, revising, or reviewing manuscripts, Emails, lectures, or proposals. He

T. Meyer $(\bowtie)$

Department of Chemistry and Biochemistry,

University of Arizona, Tucson, AZ, USA

e-mail: temeyer@u.arizona.edu did not eat lunch, but worked straight through until 5 PM when he would finally head for home. A typical day would include four meetings, sometimes less, but often more. He was involved in everything on campus. He taught a large class in biochemistry, served on the faculty senate, chaired a senate watchdog committee called the Committee of Eleven, assisted in restructuring undergraduate education, and served as faculty and research advisor to many undergraduate, graduate, and postdoc students. At various periods, he was Vice President for Research (10 years), interim Provost, Chair of Bioindustry of Southern Arizona, and Director of Arizona Research Laboratories (22 years), and maintained an active research lab throughout. In 1980, he also took a leave of absence to serve as a program director at the National Science Foundation. In 2005, he was awarded the highest academic honor at UA, that of Regents Professor. The routine was the same after his "official" retirement in 2008 .

Mike was born in Los Angeles California, March 2, 1942. Mike's father was a California State Senator from a largely Republican district and his mother a public school teacher. On his mothers side, he was descended from the Donner Party of pioneers, perhaps that is where he got his tenacity. He attended public schools, graduating at age 17, and then accepted admission to the University of The Pacific on a tennis scholarship. He was an outstanding athlete. Without knowing, I once challenged him to a game, but was thoroughly trounced. I tried again with racquetball where I was more proficient, but with the same result. I learned that Mike would not accept defeat. While an undergraduate, he had a number of summer jobs, including preparation of solid rocket fuel. While mixing the fuel, dry Santa Ana winds caused a buildup of static electricity resulting in an explosion giving him severe burns over half his body. 
After recovering from the explosion at age 21, he was among the first Chemistry graduate students at the newly formed campus of the University of California at San Diego located at the old Camp Matthews Marine Corps base in La Jolla. As a first-year student, he worked in Sverdrup Hall on the campus of Scripps Institution of Oceanography, which was allied with UCSD and where it was not uncommon for students to house their surfboards or fishing poles in the lab or hallway. Mike was no exception to this practice as he loved surf fishing. The nearby racetrack at Del Mar allowed him to engage in another interest, horseracing. In his second year, his class moved to Bonner Hall in the newly completed Revelle College up the hill from Scripps. It was a very exciting time with several Nobel laureates on campus and a cadre of well-renowned scientists. The Vietnam War led to major unrest on campus with many students and even some faculty calling for boycotts and violent action, nevertheless it had little effect on research. Torrey Pines Golf Course had a much greater impact on his life.

Mike chose Martin Kamen, an amazing scientist, as advisor. A year later, I joined the Kamen lab, quickly learning that Martin could think faster than anyone I had ever met and had a broad knowledge in all areas of science as well as being an extremely accomplished musician with a great sense of humor. In 1940, Martin, together with Sam Rubin, discovered carbon 14, perhaps the most useful of all radioactive isotopes considering that there are more papers published on its use than for any other isotope (Kamen, Ann Rev Biochem 55:1-36, 1986). Many had doubted that ${ }^{14} \mathrm{C}$ existed at all or that it would have such a long halflife. This discovery was deserving of a Nobel Prize, in fact Willard Libby was given the Nobel Prize for the radiocarbon dating method using ${ }^{14} \mathrm{C}$ in 1960 and Melvin Calvin was given the prize in 1961 for tracing the path of carbon in photosynthesis using ${ }^{14} \mathrm{C}$. But Kamen's discovery was made during the war years and at a time that he was labeled a possible information leak due to his gregarious nature and associations with leftists. It took him more than 10 years to clear his name and regain his passport.

Martin had another claim to fame, although not so dramatic as the discovery of ${ }^{14} \mathrm{C}$, in that he and Leo Vernon discovered cytochrome $c_{2}$, a homolog of mitochondrial cytochrome c, in the non-sulfur purple bacterium, Rhodospirillum rubrum, which we now know has an important role in bacterial photosynthesis and respiration. They also discovered cytochrome $\mathrm{c}^{\prime}$, one of the most commonly occurring bacterial cytochromes, which to this day has an unknown functional role. Martin had a very capable team in his lab including Bob Bartsch who added Chromatium vinosum high potential iron-sulfur protein (HiPIP) and flavocytochrome $c$ (FCSD) to their list of discoveries. It was eventually learned that HiPIP has the same role as electron donor to reaction center in purple sulfur bacteria as does cytochrome $c_{2}$ in non-sulfur purple bacteria and that FCSD functions as a sulfide dehydrogenase.

It was against this backdrop that Mike began work in the Kamen lab with guidance from Bob Bartsch. He characterized the interaction of the $C$. vinosum tetraheme reaction center cytochrome c (PufC) with the special pair bacteriochlorophyll at a time when it was believed to be two separate cytochromes. Furthermore, he investigated the effect of redox potential on the reaction. It was not until Steve Kennel came to the lab and solubilized the membrane bound cytochrome with detergent and purified it that it could be shown to have four hemes in a single peptide chain. Mike's true interest was in the kinetics of biochemical reactions. After earning his $\mathrm{PhD}$ in 1967, he went on to postdoctoral training with Quentin Gibson at Cornell University in New York. There, he continued studying protein interactions using a new technique, stopped-flow spectroscopy, which allowed measurement of binding of carbon monoxide to cytochrome $\mathrm{c}^{\prime}$ on the millisecond time scale. Mike continued his studies with stopped-flow for the next 20 years.

At age 27, Mike came to the University of Arizona as an assistant professor of chemistry. At that time, he began to develop new interests, in visual pigment and muscle contraction, but continued his study of bacterial cytochromes and photosynthesis. He served as thesis advisor to more than 20 masters and $\mathrm{PhD}$ students primarily studying the mechanism of binding and oxidation/reduction of proteins and small molecules. I came over in the mid-1970 s to collaborate with him on the binding of nucleophiles to FCSD, which is very reactive due to the unusually high redox potential of the flavin. The experiments were highly successful and Mike eventually offered me a permanent position at the University of Arizona where we wrote a grant proposal to study FCSD in more detail. The arrangement proved fruitful and it was also at this time that we engaged in a highly successful collaboration with Gordon Tollin, a well-known expert on flavins at the University of Arizona who had developed laser flash photolysis to study the kinetics of electron transfer reactions on a faster time domain. For both of us, these were our most productive research years. It was Mike's firm belief that understanding the mechanism of electron transfer required knowledge of protein structure. Thus, we developed collaborations with Richard Ambler and Jos Van Beeumen who studied amino acid sequences and evolution of cytochromes and other electron transfer proteins, with Hazel Holden, Libby Getzoff, Noritake Yasuoka, and Scott Mathews, who determined the crystal structures of cytochrome $c_{2}$, HiPIP, photoactive yellow protein, cytochrome $\mathrm{c}^{\prime}$, and FCSD.

Mikes research accomplishments include close to 300 publications. Much of his career was devoted to the study 
of cytochrome $c_{2}$, which serves as a model for mitochondrial reactions. It was known that the surface charge distribution of mitochondrial cytochrome $\mathrm{c}$ was important in its interactions with reaction partners, but the details of that interaction were largely unknown. It was through sitedirected mutagenesis in work initiated during Mike Caffrey's stay that we were able to show that a ring of positively charged amino acids located on one face of the homologous cytochrome $c_{2}$ were necessary for this interaction, whether it was with complementary negative charges on the cytochrome $b_{1}$ complex, cytochrome oxidase, or photosynthetic reaction centers. This was true whether the overall charge of the protein was neutral, positive, or negative. The interaction between $c_{2}$ and reaction centers was further elaborated in collaboration with Mel Okamura's lab in La Jolla. In this way, the influence of the dipole moment, which was the preeminent theory to explain the interaction, was proved to be largely irrelevant. Through the study of the binding of imidazole to cytochrome $c_{2}$, Chantal Dumortier in our lab showed that a section of peptide chain, which we labeled "the hinge", undergoes a localized conformational change that has physiological relevance for both bacterial cytochrome $c_{2}$ and for mitochondrial cytochrome $\mathrm{c}$.

In collaboration with Sasha Tsapin and Ken Nealson, we became involved in the study of Shewanella oneidensis, representative of a group of bacteria that are capable of dissolving and reducing insoluble metal oxides using a family of multiheme cytochromes. These reactions have enormous potential for remediating heavy metal contamination of the environment. There are one to four duplicates of this pathway, that interact with a variety of heavy metals. Electrons ultimately derive from quinones, which reduce MtrA, a periplasmic decaheme cytochrome, which communicates across the outer membrane to reduce OmcA, an extracellular decaheme cytochrome, that is presumably the direct metal ion reductase. It has not yet been proven but it is thought that STC, the abundant periplasmic small tetraheme cytochrome $\mathrm{c}$, mediates between quinones and MtrA. In another aspect of the study of electron transfer in Shewanella, soluble fumarate reductase is a chimera of STC with the well-known flavoprotein reductase for which we determined the crystal structure in collaboration with Jos Van Beeumen's lab. There is also a family of these proteins, several of whose genes are associated with homologs of histidine ammonia lyase, that possibly reduce a variety of deaminated amino acids as terminal electron acceptors. Through Mike's involvement with Arizona Research Laboratories, we determined the genome sequence of Ectothiorhodospira vacuolata. This bacterium is of interest because it contains the genes for MtrA and OmcA associated with that for HiPIP, an arrangement found in several other species, which suggests that the genes discovered in Shewanella are capable of operating in both oxidizing and reducing environments.

Mike's attention was also focused on the photoactive yellow protein (PYP), which we co-discovered with Gordon Tollin and characterized extensively (Cusanovich and Meyer, Biochemistry 42:4759-4770, 2003). There are now more than 60 species known to have PYP, which are likely to have several functional roles as judged by genetic context. This unusual signaling protein changes conformation upon trans-cis isomerization of the chromophore, resulting in transient binding to reaction partners. Savitha Devanathan performed much of the early work with PYP during her stay in the lab and collaboration with Libby Getzoff proved valuable for mutagenesis and structural characterization. John Kyndt and Mike showed that in the chimeric Ppr protein, PYP/bacteriophytochrome(Bph)/histidine kinase(HK), discovered by Ze-Yu Jiang and Carl Bauer, the Bph activates the HK upon absorption of red light. However, absorption of blue light by PYP partially blocks activation of Bph and hastens its recovery. The system is only fully reversed by action of UV light. Maarten Heyn and his students in Berlin rigorously extended laser flash photolysis of PYP and published some of the most influential papers on the subject.

Mike's most recent interest was in the potential for production of algal lipids to be used as biofuels through photosynthesis. The project was initiated by Mike, Aecio D'Silva, and John Kyndt who eventually joined a large consortium headed by Kim Ogden as lead scientist at the University of Arizona and funded by the Department of Energy. The National Alliance for Advanced Biofuels and Bioproducts continues to be geared toward genetically and environmentally optimizing lipid production in the algae to exploit their tendency to shut down protein synthesis and increase lipid production when stressed by nutrient deprivation.

In 1988, Mike became Vice President for Research, which he characterized as the best job on campus. During his tenure as VPR, the University of Arizona moved up to be ranked among the top ten public universities when yearly research funding passed $\$ 280$ million. Mike listed his greatest administrative accomplishment of that time as facilitating construction of telescopes on Mt. Graham, about 100 miles east of Tucson. It was certainly his most visible accomplishment against unrelenting opposition from radicalized environmental groups. During his administration, construction of the Large Binocular Telescope on Mt. Graham was begun. It was dedicated in 2004 and with two $8.4 \mathrm{~m}$ mirrors, it is among the worlds largest and most advanced telescopes. Also part of The Mt. Graham International Observatory are the Submillimeter Telescope and the Vatican Advanced Technology Telescope. A lesser person could never have achieved what 
Mike accomplished on Mt. Graham. As VPR, Mike provided strong support for interdisciplinary programs and created the Institute for Study of Planet Earth, the department of Biomedical Engineering, the Center for Insect Science, and the department of Neural Systems, Memory, and Aging. A restructured Graduate College increased the number of graduate degrees awarded to minority students. Mike was a strong advocate of American Indian Studies and worked to improve understanding of native cultures. Furthermore, he was co-founder of the Southern Arizona Regional Science and Engineering Fair.

Mike was Director of Arizona Research Laboratories from 1988 until his passing. ARL is a large service facility that provides expertise in many technical areas, including but not limited to the Biotechnical Computing Facility, dealing with robotics and automation, data mining, and bioinformatics, The Biological Magnetic Imaging Laboratory, The Cytometry Core Facility involved in cell sorting, the Genomic Analysis and Technology Core, providing DNA sequence analysis, University Spectroscopy and Imaging Facility, providing transmission and scanning electron microscopy, Human Origins Genotyping Laboratory, providing technical support for National Geographics, genealogical reconstruction for FamilyTreeDNA, and forensic DNA reconstruction for the DNA Shoah Project, and the Arizona Proteomics Consortium, which does mass spectroscopic identification of peptides. During his tenure as director, Mike expanded these services and obtained state of the art equipment to keep them operating efficiently, a difficult task compounded by shrinking resources. Mike helped found and was head of the Bioindustry Organization of Southern Arizona and campaigned hard to attract bioindustry to the state.

Most administrators give up teaching and research, but Mike continued operating his lab successfully while VPR and eventually returned 10 years later without any indication that he had been away from full-time research activity. Moreover, he resumed teaching as if he had never missed a lecture. It was with great shock and sadness that we learned of his death of an apparent heart attack on April 12, 2010. Mike was a wonderful person to work for and had a great sense of humor. He occasionally liked to intimidate adversaries and when he was VPR had a sign on his desk that read "what part of NO don't you understand". He was not afraid to make decisions and admired aggressiveness in faculty members. He was able to overlook the faults of others provided they got results. He had many outside interests, including virtually all sports, horseback riding, archaeology, modeling, reading, history, stamps, antique weapons, the Southwest, and native cultures. It is true that life goes on, but it is not as much fun without him. 